\title{
Spin-orbital coupling effect on power factor in semiconducting transition-metal dichalcogenide monolayers
}

\author{
San-Dong Guo and Jian-Li Wang \\ Department of Physics, School of Sciences, China University of Mining and Technology, Xuzhou 221116, Jiangsu, China
}

\begin{abstract}
The electronic structures and thermoelectric properties of semiconducting transition-metal dichalcogenide monolayers $\mathrm{MX}_{2}(\mathrm{M}=\mathrm{Zr}, \mathrm{Hf}, \mathrm{Mo}, \mathrm{W}$ and $\mathrm{Pt} ; \mathrm{X}=\mathrm{S}$, Se and $\mathrm{Te})$ are investigated by combining first-principles and Boltzmann transport theory, including spin-orbital coupling (SOC). It is found that the gap decrease increases from $\mathrm{S}$ to Te in each cation group, when the SOC is opened. The spin-orbital splitting has the same trend with gap reducing. Calculated results show that SOC has noteworthy detrimental effect on p-type power factor, while has a negligible influence in n-type doping except $\mathrm{W}$ cation group, which can be understood by considering the effects of SOC on the valence and conduction bands. For $\mathrm{WX}_{2}(\mathrm{X}=\mathrm{S}$, Se and Te), the SOC leads to observably enhanced power factor in n-type doping, which can be explained by SOC-induced band degeneracy, namely bands converge. Among all cation groups, Pt cation group shows the highest Seebeck coefficient, which leads to best power factor, if we assume scattering time is fixed. Calculated results show that $\mathrm{MS}_{2}(\mathrm{M}=\mathrm{Zr}, \mathrm{Hf}, \mathrm{Mo}, \mathrm{W}$ and $\mathrm{Pt})$ have best p-type power factor for all cation groups, and that $\mathrm{MSe}_{2}(\mathrm{M}=\mathrm{Zr}$ and $\mathrm{Hf}), \mathrm{WS}_{2}$ and $\mathrm{MTe}_{2}(\mathrm{M}=\mathrm{Mo}$ and $\mathrm{Pt})$ have more wonderful $\mathrm{n}$-type power factor in respective cation group. Therefore, these results may be useful for further theoretical prediction or experimental search of excellent thermoelectric materials from semiconducting transition-metal dichalcogenide monolayers.
\end{abstract}

PACS numbers: 72.15.Jf, 71.20.-b, 71.70.Ej, 79.10.-n

Email:guosd@cumt.edu.cn

Keywords: Transition-metal dichalcogenide monolayers; Spin-orbit coupling; Power factor

\section{INTRODUCTION}

Due to the Seebeck effect and Peltier effect, the hotelectricity conversion can be achieved in thermoelectric materials to solve energy issues, and the dimensionless figure of merit[1, 2], $Z T=S^{2} \sigma T /\left(\kappa_{e}+\kappa_{L}\right)$, can characterize the efficiency of thermoelectric conversion, where $\mathrm{S}, \sigma, \mathrm{T}, \kappa_{e}$ and $\kappa_{L}$ are the Seebeck coefficient, electrical conductivity, absolute temperature, the electronic and lattice thermal conductivities, respectively. It is interesting and challenging to search for high $Z T$ materials, and one of the key parameters is power factor $\left(S^{2} \sigma\right)$, which depends on electronic structures of materials. As is well known, SOC has important effect on electronic structures of materials containing heavy element like famous topological insulators[3, 4]. Recently, the SOC has been proved to be very important for power factor calculations[5-11]. For thermoelectric material $\mathrm{Mg}_{2} \mathrm{Sn}$, when the SOC is included, the best n-type power factor is higher than the best one in p type doping, which agrees with the experimental results[12]. Therefore, it is very important to consider SOC for theoretical analysis of power factor.

The discovery of graphene leads to extensive attention on two-dimensional (2D) nanostructures due to their unusual physical, mechanical and chemical properties, 2D transition-metal dichalcogenides (TMDs) of which have potential application in nanoelectronics and nanophotonics[13-21]. The physical and chemical properties of 2D-TMDs can be tuned by strain, applied electric field, controlling the composition and functionalizing[22-28]. Thermoelectric properties of low-

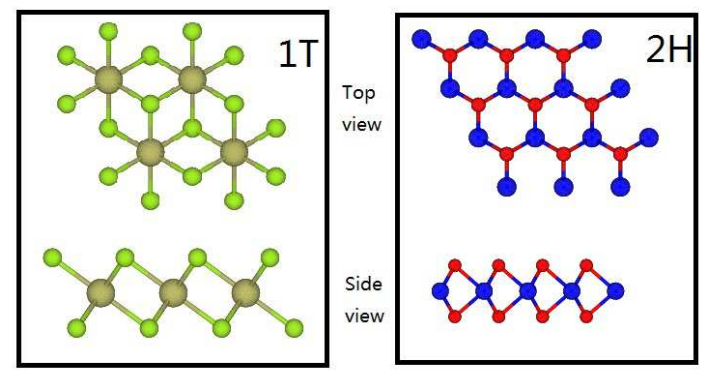

FIG. 1. (Color online) Two crystal structures of single-layer $\mathrm{MX}_{2}: 1 \mathrm{~T}$ structure and $2 \mathrm{H}$ structure.

dimensional materials have been a hotspot for their applications into high-performance thermoelectric devices, such as $\mathrm{Bi}_{2} \mathrm{Te}_{3}$ nanowire, 2D-phosphorene and silicene[29-33]. The thermoelectric properties related with low-dimensional TMDs, including few layers, monolayers and nanotubes, have been widely investigated[3438]. However, the SOC is neglected in these theoretical calculation of thermoelectric properties related with lowdimensional TMDs. Recently, we prove that SOC has very obvious effect on p-type power factor for $\mathrm{MoS}_{2}[39]$.

Here, we systematically investigate the electronic structures and thermoelectric properties of semiconducting TMD monolayers $\mathrm{MX}_{2}(\mathrm{M}=\mathrm{Zr}, \mathrm{Hf}, \mathrm{Mo}, \mathrm{W}$ and $\mathrm{Pt}$; $\mathrm{X}=\mathrm{S}$, Se and Te) by first-principles calculations and semiclassical Boltzmann transport theory within the generalized gradient approximation (GGA) plus SOC. It is found that both SOC-induced gap reducing and spin-orbital splitting increase from $\mathrm{S}$ to Te in each cation group. Cal- 
TABLE I. The optimized lattice constant $a(\AA)$; the calculated gap values with GGA $E_{1}(\mathrm{eV})$ and GGA+SOC $E_{2}(\mathrm{eV})$; $E_{1}-E_{2}(\mathrm{eV})$; spin-orbit splitting $\Delta(\mathrm{eV})$ at the $\Gamma$ point with $1 \mathrm{~T}$ structure or the $\mathrm{K}$ point with $2 \mathrm{H}$ structure near the Fermi level in the valence bands. These values in the parentheses are else theoretical values in ref.[51, 52].

\begin{tabular}{cccccc}
\hline \hline Name & $a$ & $E_{1}$ & $E_{2}$ & $E_{1}-E_{2}$ & $\Delta$ \\
\hline \hline $\mathrm{ZrS}_{2}$ & 3.68 & $1.16(1.19)$ & 1.12 & 0.04 & 0.09 \\
\hline $\mathrm{ZrSe}_{2}$ & 3.80 & $0.50(0.50)$ & 0.35 & 0.15 & 0.28 \\
\hline $\mathrm{HfS}_{2}$ & 3.64 & $1.22(1.27)$ & 1.16 & 0.06 & 0.13 \\
\hline $\mathrm{HfSe}_{2}$ & 3.76 & $0.59(0.61)$ & 0.42 & 0.17 & 0.32 \\
\hline $\mathrm{MoS}_{2}$ & 3.18 & $1.70(1.68)$ & 1.63 & 0.07 & $0.15(0.148)$ \\
\hline $\mathrm{MoSe}_{2}$ & 3.32 & $1.44(1.45)$ & 1.34 & 0.10 & $0.18(0.184)$ \\
\hline $\mathrm{MoTe}_{2}$ & 3.55 & $1.09(1.08)$ & 0.96 & 0.13 & 0.21 \\
\hline $\mathrm{WS}_{2}$ & 3.18 & $1.86(1.82)$ & 1.60 & 0.26 & $0.42(0.430)$ \\
\hline $\mathrm{WSe}_{2}$ & 3.32 & $1.56(1.55)$ & 1.28 & 0.28 & $0.46(0.466)$ \\
\hline $\mathrm{WTe}_{2}$ & 3.55 & $1.09(1.07)$ & 0.78 & 0.31 & 0.48 \\
\hline $\mathrm{PtS}_{2}$ & 3.57 & $1.76(1.81)$ & 1.73 & 0.03 & 0.21 \\
\hline $\mathrm{PtSe}_{2}$ & 3.75 & $1.37(1.41)$ & 1.20 & 0.17 & 0.34 \\
\hline $\mathrm{PtTe}_{2}$ & 4.02 & $0.77(0.79)$ & 0.38 & 0.39 & 0.49 \\
\hline \hline
\end{tabular}

culated results show that SOC not only can reduce power factor in p-type doping, but also can enhance one in ntype doping, especially for $\mathrm{W}$ cation group. These can be understood by considering their energy band structures. It is found that $\mathrm{PtX}_{2}(\mathrm{X}=\mathrm{S}$, Se and Te) may have more excellent thermoelectric properties due to the very high Seebeck coefficients.

The rest of the paper is organized as follows. In the next section, we shall briefly describe computational details. In the third section, we shall present the electronic structures and thermoelectric properties of semiconducting TMD monolayers. Finally, we shall give our discussions and conclusion in the fourth section.

\section{COMPUTATIONAL DETAIL}

First-principles calculations of semiconducting TMD monolayers are performed using density functional theory[40] within full-potential linearized augmentedplane-waves method, as implemented in the package WIEN2k [41]. The GGA of Perdew, Burke and Ernzerhof (PBE) [42] is used for the exchange-correlation potential. The full relativistic effects are calculated with the Dirac equations for core states, and the scalar relativistic approximation is used for valence states [43-45]. The SOC was included self-consistently by solving the radial Dirac equation for the core electrons and evaluated by the second-variation method[46]. We use $6000 \mathrm{k}$-points in the first Brillouin zone for the self-consistent calculation. We make harmonic expansion up to $l_{\max }=10$ in each of the atomic spheres, and the plane-wave cut-
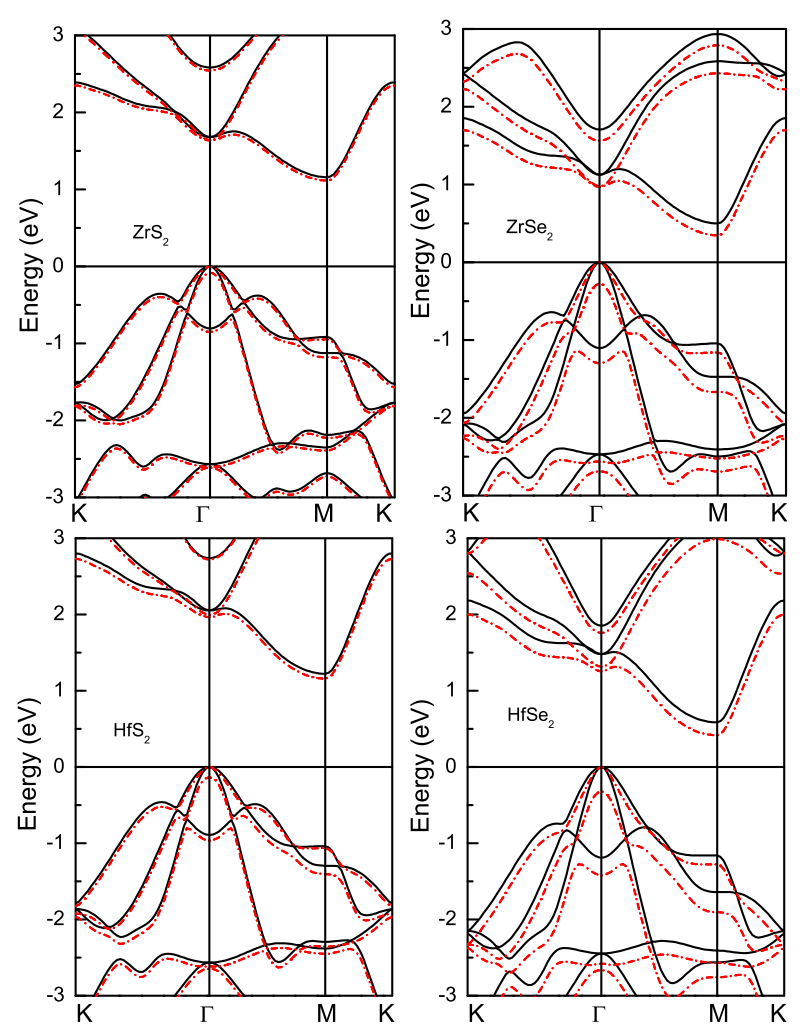

FIG. 2. (Color online) The energy band structures of monolayer $\mathrm{MX}_{2}(\mathrm{M}=\mathrm{Zr}$, Hf; $\mathrm{X}=\mathrm{S}$, Se) by using GGA (Black solid lines) and GGA+SOC (Red short dash dot lines).

off is determined by $\mathrm{R}_{\mathrm{mt}} * \mathrm{k}_{\max }=8$. The self-consistent calculations are considered to be converged when the integration of the absolute charge-density difference between the input and output electron density is less than $0.0001|e|$ per formula unit, where $e$ is the electron charge. Transport calculations are performed using semiclassical Boltzmann transport theory and the rigid band approach within the constant scattering time approximation (CSTA) as implemented in BoltzTrap[47] (Note: the parameter LPFAC can not choose the default value 5, and should choose larger value. Here, we choose LPFAC value for 20.), which has been applied successfully to several materials[48-50]. To enable accurate Fourier interpolation of the Kohn-Sham eigenvalues, we use 90000 k-points in the first Brillouin zone for the energy band calculation.

\section{MAIN CALCULATED RESULTS AND ANALYSIS}

The single-layer $\mathrm{MX}_{2}$ contains three atomic sublayers with the metal atom $\mathrm{M}$ in the center sublayer, while $\mathrm{X}$ atoms locate in the top and bottom sublayers. The different stacking of top and bottom X sublayers leads to two crystal structures, namely $1 \mathrm{~T}$ structure $(\mathrm{M}=\mathrm{Ti}, \mathrm{V}$, $\mathrm{Zr}$, Hf and $\mathrm{Pt})$ and $2 \mathrm{H}$ structure $(\mathrm{M}=\mathrm{Nb}, \mathrm{Mo}$, Ta and 

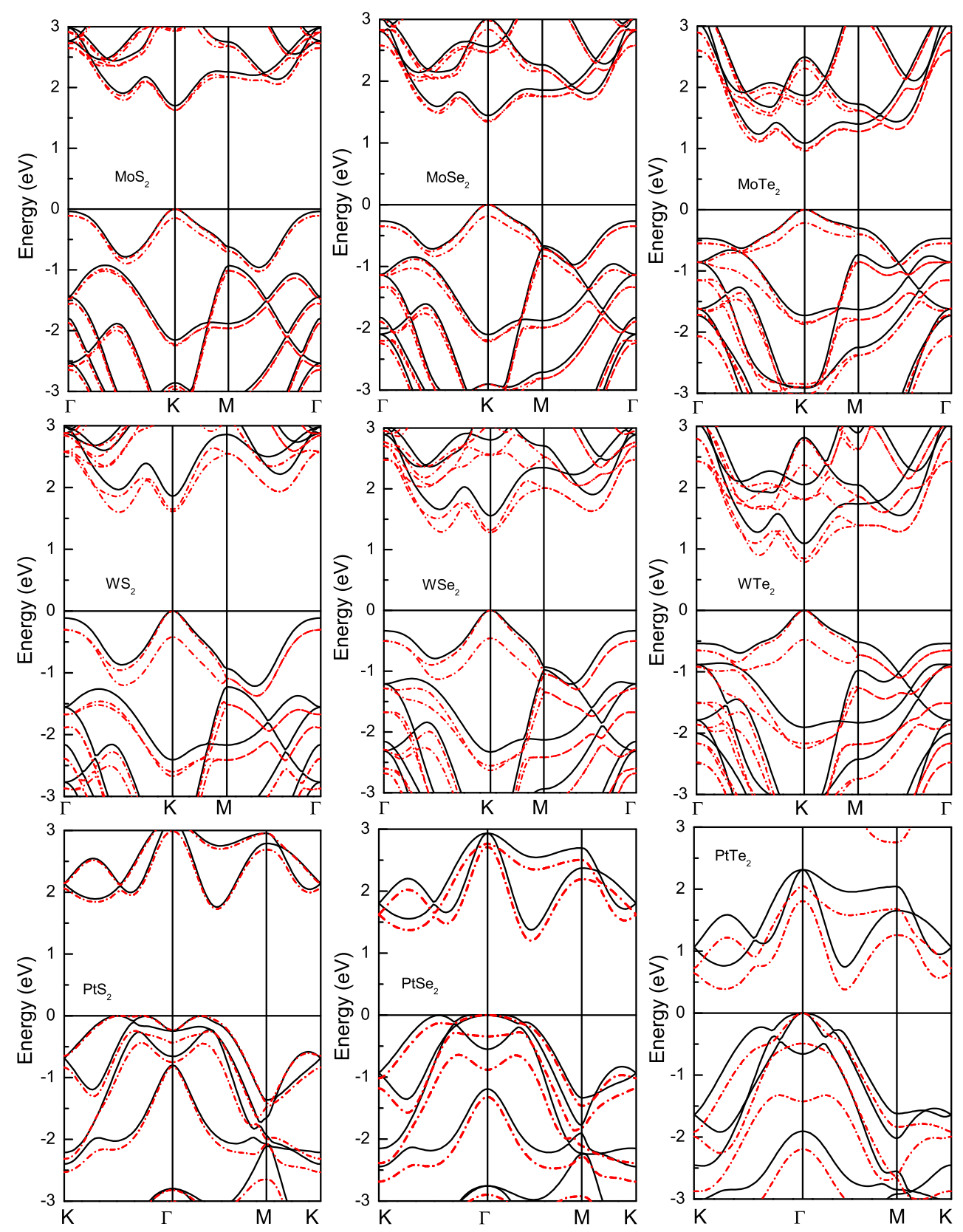

FIG. 3. (Color online) The energy band structures of monolayer $\mathrm{MX}_{2}(\mathrm{M}=\mathrm{Mo}, \mathrm{W}, \mathrm{Pt}$; X=S, Se, Te) by using GGA (Black solid lines) and GGA+SOC (Red short dash dot lines).

W), which are shown in Figure 1. The ionicity of $\mathrm{MX}_{2}$ leads to two distinctive structures, and the $1 \mathrm{~T}$ structure supports higher ionicity, which is due to longer distance between $\mathrm{X}$ atoms of the top and bottom sublayers. In Ref.[51] , H. L. Zhuang et al. predict that 27 single-layer $\mathrm{MX}_{2}$ can be fabricated from bulk crystals due to small formation energies (Some of them have been achieved experimentally, such as $\mathrm{MoS}_{2}, \mathrm{MoSe}_{2}$ and $\left.\mathrm{WSe}_{2}.\right), 13 \mathrm{MX}_{2}$ of which are semiconductors. Because good thermoelectric materials are usually narrow-gap semiconductors, we focus on semiconducting TMD monolayers $\mathrm{MX}_{2}(\mathrm{M}=\mathrm{Zr}$,
Hf, Mo, W and Pt; $\mathrm{X}=\mathrm{S}$, Se and Te). The crystal structure of single-layer $\mathrm{MX}_{2}$ is built with the vacuum region of $18 \AA$ to avoid spurious interaction. The optimized lattice constants $a[51]$ are used to do our DFT calculations, which are listed in Table I.

We investigate their electronic structures by using GGA and GGA+SOC, and plot their energy band structures in Figure 2 and Figure 3 (Note: The high-symmetry path $\mathrm{K}-\Gamma-\mathrm{M}-\mathrm{K}$ is chosen for $1 \mathrm{~T}$ structure, and $\Gamma-\mathrm{K}-\mathrm{M}-$ $\Gamma$ for $2 \mathrm{H}$ structure.). Both GGA and GGA+SOC show that $\mathrm{MoX}_{2}$ and $\mathrm{WX}_{2}$ are direct band gap semiconductors 

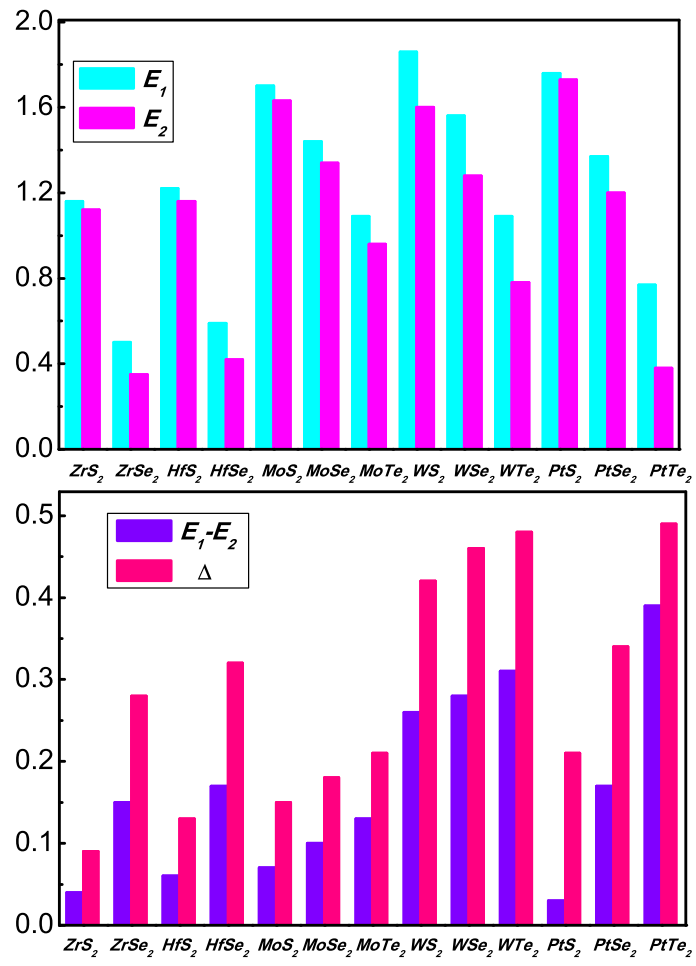

FIG. 4. (Color online) Top panel: the calculated gap values with GGA $E_{1}(\mathrm{eV})$ and GGA+SOC $E_{2}(\mathrm{eV})$; Bottom panel: $E_{1}-E_{2}(\mathrm{eV})$ and spin-orbit splitting $\Delta(\mathrm{eV})$ at the $\Gamma$ point with $1 \mathrm{~T}$ structure or the $\mathrm{K}$ point with $2 \mathrm{H}$ structure near the Fermi level in the valence bands.

with the conduction band minimum $(\mathrm{CBM})$ and valence band maximum (VBM) at the K point (Note: When the $\mathrm{SOC}$ is included, $\mathrm{WS}_{2}$ is a indirect semiconductor). The $\mathrm{ZrX}_{2}$ and $\mathrm{HfX}_{2}$ are indirect band gap semiconductors with the VBM at the $\Gamma$ point and CBM at the M point. The VBM appears at the $\Gamma$ point, while the CBM is between the $\Gamma$ and $\mathrm{K}$ points for $\mathrm{PtSe}_{2}$ and $\mathrm{PtTe}_{2}$. The VBM of $\mathrm{PtS}_{2}$ is between the $\Gamma$ and $\mathrm{K}$ points, while the CBM appears between the $\Gamma$ and $M$ points. The GGA gaps, GGA+SOC gaps and the differences between them are listed in Table I. Calculated GGA gaps are consistent with else theoretical values[34, 51]. To clearly see the gap trend, the related gap values also are plotted in Figure 4. It is found that the gap of $\mathrm{MX}_{2}$ decreases from $\mathrm{S}$ to Te with the same $\mathrm{M}$, while the difference between GGA and GGA+SOC gaps gradually increases. The larger gap decrease means the larger movement of conduction bands toward low energy, which reflects the SOC effects on the conduction bands. The SOC effects on the valence bands near Fermi level can be described by spin-orbit splitting at the $\Gamma$ point with $1 \mathrm{~T}$ structure or the $\mathrm{K}$ point with $2 \mathrm{H}$ structure near the Fermi level in the valence bands, which are listed in Table I and plotted in Figure 4. Our GGA spin-orbit splitting values agree with else calculated ones[52, 53]. From $\mathrm{S}$ to Te, the spin-orbital splitting value increases in each cation group. These data show that SOC produces more obvious effects on the valence bands than the conduction bands.

The semi-classic transport coefficients are calculated within CSTA Boltzmann theory. Calculating scattering time from first-principles calculations is challenging due to the complexity of various carrier scattering mechanisms. To mimic the doping effects on the transport coefficients, the rigid band approach is used, and only the Fermi level is shifted to change the doping level. If the doping level is low, the rigid band approximation is reasonable, which has been widely used for theoretical study of thermoelectric materials[47, 54-56], and the calculated transport coefficients agree well with experimental results. The Seebeck coefficient $S$, electrical conductivity with respect to scattering time $\sigma / \tau$ and power factor with respect to scattering time $\mathrm{S}^{2} \sigma / \tau$ as a function of doping level at the temperature of $300 \mathrm{~K}$ by using GGA and GGA+SOC are shown in Figure 5. The n-type doping (negative doping levels) with the negative Seebeck coefficient is related with conduction bands, while p-type doping (positive doping levels) with the positive Seebeck coefficient is connected with valence bands. When the Fermi level locates the middle of band gap, the Seebeck coefficient has a very large value, but low electrical conductivity due to low carrier concentration leads to very small power factor. As the Fermi level move into conduction bands or valence bands (change the doping level), the electrical conductivity increases and Seebeck coefficient decreases, and the power factor reaches its maximum at certain doping level.

Firstly, we consider SOC effects on transport coefficients $\mathrm{S}$, which is independent of scattering time $\tau$, and can be directly compared with experimental results. It is found that SOC has a detrimental influence on S (absolute value) in p-type doping, but has a negligible effect in n-type doping except $\mathrm{WX}_{2}$. These can be explained by SOC effects on the valence and conduction bands. The SOC removes the valence band degeneracy, which reduces slope of density of states (DOS) of valence bands near the energy gap, and leads to reduced Seebeck coefficient. Here, we only plot DOS of $\mathrm{MoS}_{2}$ and $\mathrm{WS}_{2}$ calculated with GGA and GGA+SOC as representative in Figure 6. Calculated results show 2D DOS near the Fermi level, which is close to a step function. The slope of DOS of valence bands near the energy band gap decreases, when SOC is included, which lead to reduced S. Similar 2D-like DOS also can be found in bulk materials $\mathrm{PbX}(\mathrm{X}=\mathrm{S}$, Se and $\mathrm{Te})[57]$ and $\mathrm{BiTeI}[58]$. The SOC makes the conduction bands nearly overall move toward the Fermi level for $\mathrm{MX}_{2}(\mathrm{M}=\mathrm{Zr}$, Hf, Mo and Pt), and the outlines of conduction bands have little change, which leads to weak SOC effects on S. However, the SOC has obvious influences on the conduction bands near the Fermi level for $\mathrm{WX}_{2}$. The SOC-induced splitting between $\Gamma$ and $\mathrm{K}$ points for conduction bands is very remarkable, 

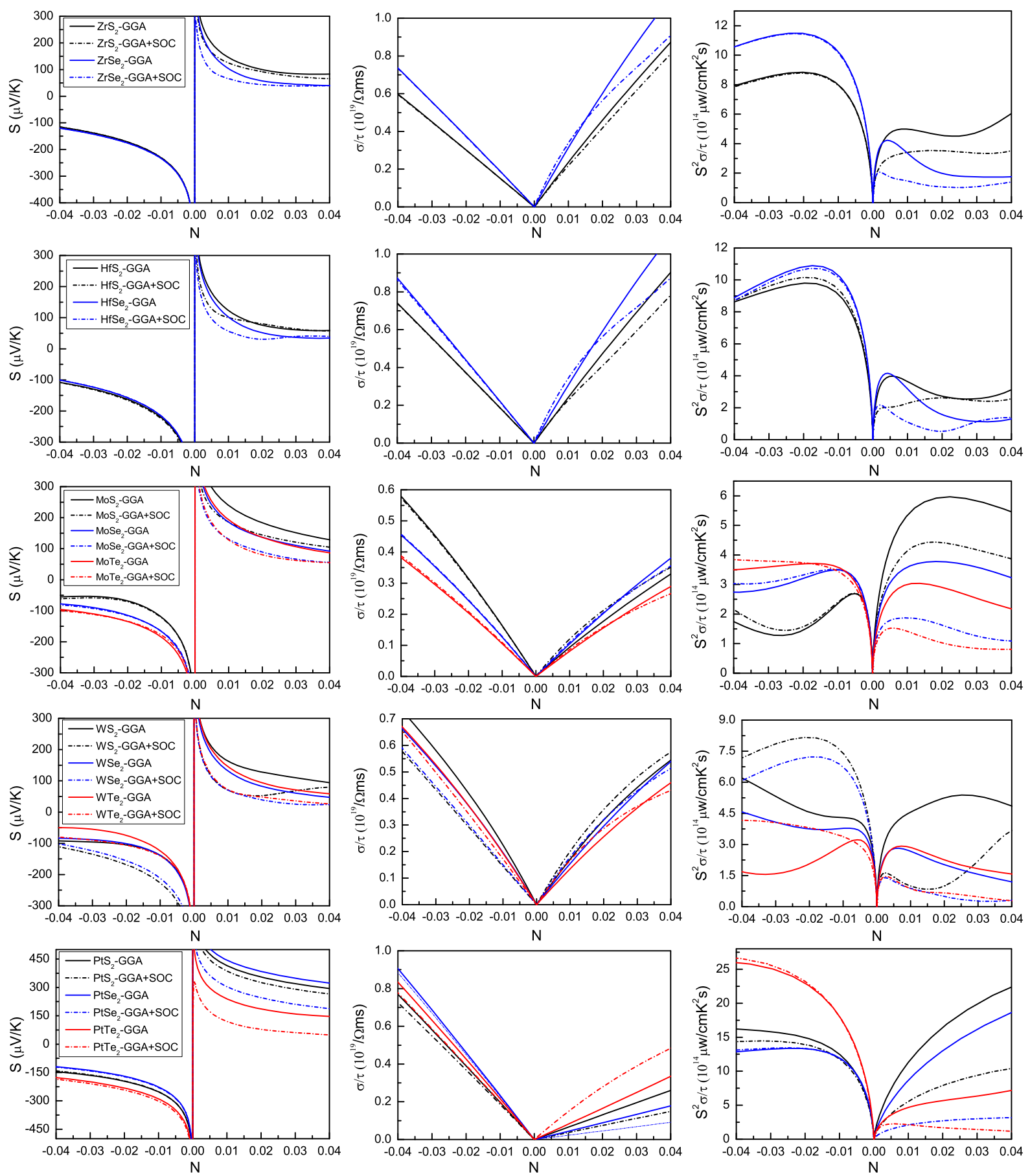

FIG. 5. (Color online) At $\mathrm{T}=300 \mathrm{~K}$, transport coefficients, including Seebeck coefficient $\mathrm{S}$ (Left), electrical conductivity with respect to scattering time $\sigma / \tau$ (Middle) and power factor with respect to scattering time $\mathrm{S}^{2} \sigma / \tau$ (Right), as a function of doping level calculated with GGA (solid lines) and GGA+SOC (short dash dot lines). The doping level is defined as electrons (minus value) or holes (positive value) per unit cell.

which leads to near degeneracy between conduction band extremum along $\Gamma-\mathrm{K}$ line and one at $\mathrm{K}$ point, especially for $\mathrm{WS}_{2}$ and $\mathrm{WSe}_{2}$. The SOC-induced band degeneracy, namely bands converge, can enhance the S. Similar bands converge can be induced in $\mathrm{Mg}_{2} \mathrm{Sn}$ by pressure or doping[10,59]. Figure 6 show that SOC can lead to
2D more-like DOS of conduction bands near the Fermi level for $\mathrm{WS}_{2}$. Secondly, the SOC has little effects on $\sigma / \tau$ for $\mathrm{MX}_{2}(\mathrm{M}=\mathrm{Zr}$, Hf and $\mathrm{Mo})$ in n-type doping, but has a observable effects for $\mathrm{MX}_{2}(\mathrm{M}=\mathrm{W}$ and $\mathrm{Pt})$. For p-type doping, the SOC influences on $\sigma / \tau$ are more obvious with respect to ones in n-type doping. These can 


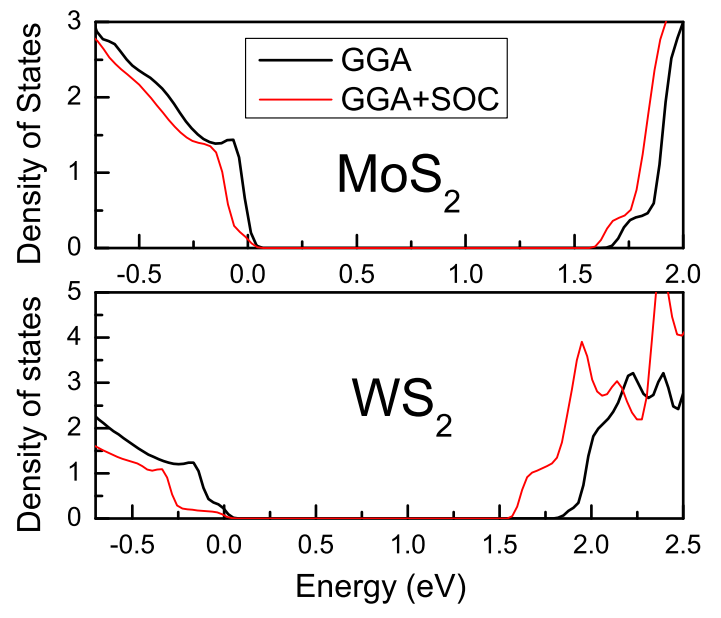

FIG. 6. (Color online) The density of states of $\mathrm{MoS}_{2}$ and $\mathrm{WS}_{2}$ calculated with GGA and GGA+SOC.

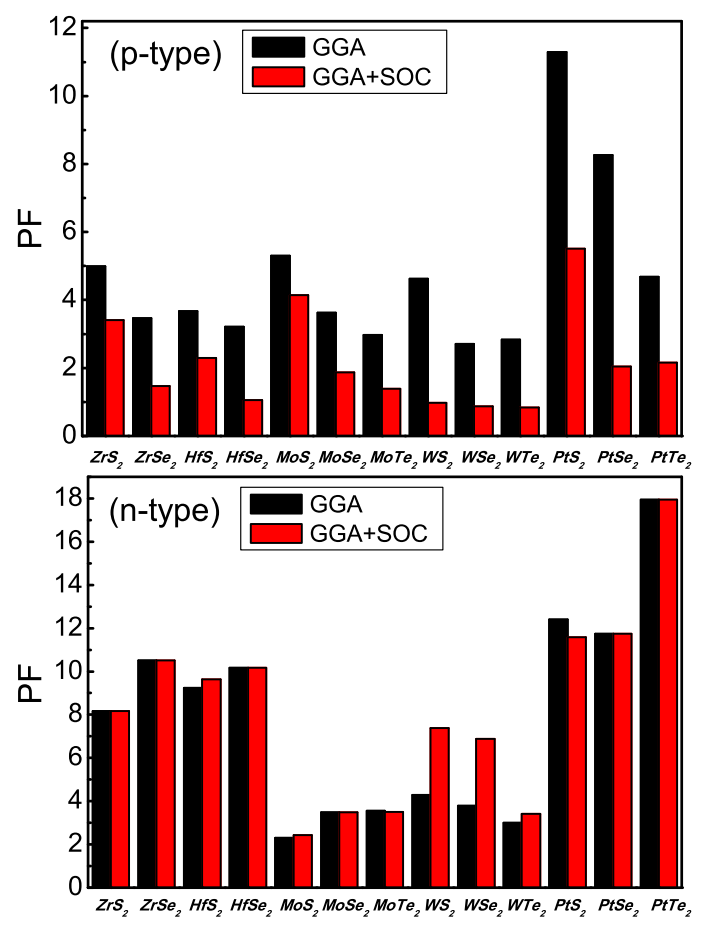

FIG. 7. (Color online) The power factors with the doping level 0.01 electrons (n-type) or holes (p-type) per unit cell by using GGA and GGA+SOC at T=300 K.

be understood by SOC-induced band localization or delocalization.

Finally, the SOC effects on power factor are considered, which is a comprehensive physical quantity for the electrical performance of thermoelectric materials. Due to power factor being proportional to $\mathrm{S}$ and $\sigma$, the SOC has a remarkable detrimental influence on power factor in p-type doping, while has a weak influence but $\mathrm{WX}_{2}$ for $\mathrm{n}$-type. The power factors of $\mathrm{WX}_{2}$ can be significantly improved due to the openness of SOC. To clearly see the SOC effects on power factor, the power factors with the doping level 0.01 electrons or holes per unit cell by using GGA and GGA+SOC at T=300 K are plotted in Figure 7. Although the scattering time $\tau$ is unknown, comparison of relative power factor values among these TMD monolayers $\mathrm{MX}_{2}$ may be useful for experimental guidance on searching the excellent thermoelectric materials. For Zr, Hf, W and Pt series, the n-type doping has better power factors than p-type doping. For $\mathrm{MoX}_{2}$, the p-type power factor of $\mathrm{MoS}_{2}$ is larger than one in ntype doping, while it is opposite for $\mathrm{MoSe}_{2}$ and $\mathrm{MoTe}_{2}$. These can be easily observed from Figure 7 . If we assume the scattering time $\tau$ is constant for $\mathrm{MX}_{2}$, the $\mathrm{Pt}$ series have larger power factor due to the larger Seebeck coefficient S. It is worth noting that local Rashba spin polarization and spin-layer locking have been observed in monolayer $\mathrm{PtSe}_{2}$, which can realize electrically tunable spintronics[60]. For each cation group, we summarize the best power factor for both n-type and p-type, and are shown in Figure 8. It is found that $\mathrm{MS}_{2}$ has best power factor in p-type doping for all cation groups. For n-type, $\mathrm{MSe}_{2}(\mathrm{M}=\mathrm{Zr}$ and $\mathrm{Hf}), \mathrm{WS}_{2}$ and $\mathrm{MTe}_{2}(\mathrm{M}=\mathrm{Mo}$ and $\mathrm{Pt}$ ) have more excellent power factor in respective cation group.

\section{DISCUSSIONS AND CONCLUSION}

As is well known, the SOC removes the band degeneracy by spin-orbit splitting, which can modify the outlines of energy bands. These SOC effects can lead to remarkable influence on Seebeck coefficient, and further affect the power factor. The SOC-induced detrimental influence on power factor in $\mathrm{Mg}_{2} \mathrm{Sn}[5,10]$ and half-Heusler $\mathrm{ANiB}(\mathrm{A}=\mathrm{Ti}, \mathrm{Hf}, \mathrm{Sc}, \mathrm{Y} ; \mathrm{B}=\mathrm{Sn}, \mathrm{Sb}, \mathrm{Bi})[6]$, especially for p-type doping, has been observed. For monolayer $\mathrm{MX}_{2}$, SOC can reduce power factor for p-type in the considered doping range, but also can improve one in n-type doping for some of them, especially for $\mathrm{WX}_{2}$. The SOC also can lead to the conversion of best power factor between $n-$ and p-type doping. For example, at the absence of SOC, the p-type has better power factor for $\mathrm{PtS}_{2}$. However, including SOC, the n-type doping shows more excellent power factor. So, it is very important for power factor calculations to include SOC for semiconducting TMD monolayers $\mathrm{MX}_{2}$.

Symmetry driven degeneracy, low-dimensional electronic structures and accidental degeneracies[61] can lead to enhanced power factor. Here, SOC-induced degeneracy of conduction band extremum produces significantly improved power factor for $\mathrm{WX}_{2}(\mathrm{~W}=\mathrm{S}$ and $\mathrm{Se})$. In fact, strain is a very effective way to tune the electronic structures of materials, which can achieve improved power factor. The electronic structures of monolayer $\mathrm{MX}_{2}$ have sensitive strain dependence, which provides a platform to realize higher power factor. In Ref.[39], the first-principle 


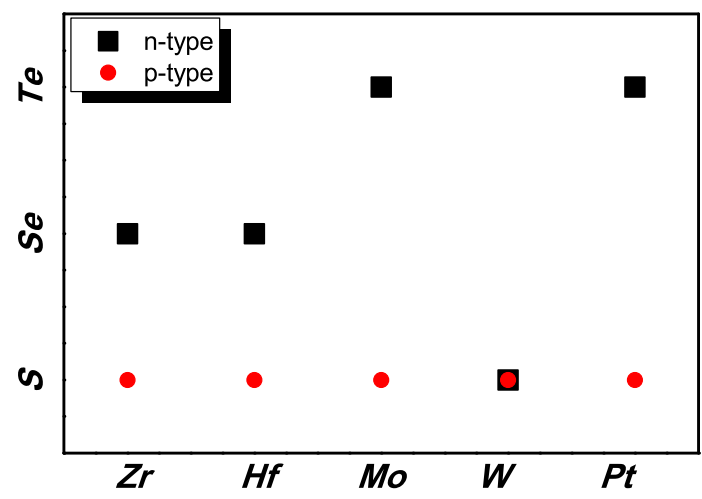

FIG. 8. (Color online) The best n- and p-type power factor for each cation group by using GGA+SOC at T=300 K.

calculations predict that both compressive and tensile strain at the critical strain of direct-indirect gap transition can induce the accidental degeneracies for $\mathrm{MoS}_{2}$, which can produce more excellent power factor in certain doping range. For Pt cation group, the outlines of valence bands near the Fermi level are very distinct. However, the outline of $\mathrm{PtS}_{2}$ can be attained from recentlysynthesized $\mathrm{PtSe}_{2}[62]$ by compressive strain, and the one of $\mathrm{PtTe}_{2}$ also can be achieved by tensile strain (These calculation have been tested). Therefore, It is possible to realize higher power factor for semiconducting monolayer $\mathrm{MX}_{2}$ by strain tuning.

In summary, we investigate electronic structures and thermoelectric properties of semiconducting TMD monolayers using GGA+SOC, based mainly on the reliable first-principle calculations and Boltzmann transport theory. It is found that including SOC is very crucial for power factor calculations, due to remarkable SOC influences on the energy band structures of TMD monolayers. Calculated results show that Pt series may be potential thermoelectric materials due to the large Seebeck coefficient S. The present work is useful for further theoretical calculations and experimental guidance on searching the excellent thermoelectric materials from TMD monolayers.

This work is supported by the National Natural Science Foundation of China (Grant No.11404391). We are grateful to the Advanced Analysis and Computation Center of CUMT for the award of CPU hours to accomplish this work.

[1] S. Chen and Z. F. Ren, Mater. Today 16, 387 (2013).

[2] Y. Pei, X. Shi, A. LaLonde, H. Wang, L. Chen and G. J. Snyder, Nature 473, 66 (2011).

[3] M. Z. Hasan and C. L. Kane, Rev. Mod. Phys. 82, 3045 (2010).

[4] L. Fu, C. L. Kane, and E. J. Mele, Phys. Rev. Lett. 98, 106803 (2007).
[5] K. Kutorasinski, B. Wiendlocha, J. Tobola and S. Kaprzyk, Phys. Rev. B 89, 115205 (2014).

[6] S. D. Guo, J. Alloy. Compd. 663, 128 (2016).

[7] P. Larson, S. D. Mahanti, and M. G. Kanatzidis, Phys. Rev. B 61, 8162 (2000).

[8] T. J. Scheidemantel, C. Ambrosch-Draxl, T. Thonhauser, J. V. Badding, and J. O. Sofo, Phys. Rev. B 68, 125210 (2003).

[9] S. J. Youn and A. J. Freeman, Phys. Rev. B 63, 085112 (2001).

[10] S. D. Guo and J. L. Wang, RSC Adv. 6, 31272 (2016).

[11] N. Singh and U. Schwingenschlögl, Phys. Status Solidi RRL 08, 805 (2014).

[12] V. K. Zaitsev, M. I. Fedorov, E. A. Gurieva, I. S. Eremin, P. P. Konstantinov, A. Y. Samunin and M. V. Vedernikov, Phys. Rev. B 74, 045207 (2006).

[13] A. H. Castro Neto, F. Guinea, N. M. R. Peres, K. S. Novoselov and A. K. Geim, Rev. Mod. Phys. 81, 109 (2009).

[14] Gian G. Guzmán-Verri and L. C. Lew Yan Voon, Phys. Rev. B 76, 075131 (2007).

[15] E. Bianco, S. Butler, S. S. Jiang, O. D. Restrepo, W. Windl and J. E. Goldberger, ACS Nano 7, 4414 (2013).

[16] M. Chhowalla, H. S. Shin, G. Eda, L. J. Li, K. P. Loh and H. Zhang, Nat. Chem. 5263 (2013).

[17] X. D. Xu, W. Yao, D. Xiao and T. F. Heinz, Nature Phys. 10, 343 (2014).

[18] P. Rastogi, S. Kumar, S. Bhowmick, A. Agarwal and Y. S. Chauhan, J. Phys. Chem. C 118, 30309 (2014).

[19] K. F. Mak, C. Lee, J. Hone, J. Shan, and T. F. Heinz, Phys. Rev. Lett. 105, 136805 (2010).

[20] A. Splendiani, L. Sun, Y. Zhang, T. Li, J. Kim, C. Y. Chim, G. Galli and F. Wang, Nano Lett. 10, 1271 (2010).

[21] B. Radisavljevic, A. Radenovic, J. Brivio, V. Giacometti and A. Kis, Nature Nanotechnology 6, 147 (2011).

[22] H. Pan, and Y. W. Zhang, J. Phys. Chem. C 116, 1175 (2012).

[23] H. Shi, H. Pan, Y. W. Zhang, and B. I. Yakobson, Phys. Rev. B 88, 205305 (2013).

[24] C. Ataca and S. Ciraci, J. Phys. Chem. C 115, 13303 (2011).

[25] S. Bhattacharyya and A. K. Singh, Phys. Rev. B 86, 075454 (2012).

[26] E. Scalise, M. Houssa, G. Pourtois, V. Afanas'ev and A. Stesmans, Nano Res. 5, 43 (2012).

[27] W. S. Yun, S. W. Han, S. C. Hong, I. G. Kim and J. D. Lee, Phys. Rev. B 85, 033305 (2012).

[28] Q. Liu, L. Li, Y. Li, Z. Gao, Z. Chen and J. Lu, J. Phys. Chem. C 116, 21556 (2012).

[29] J. F. Li, W. S. Liu, L. D. Zhao and M. Zhou, NPG Asia Mater. 2, 152 (2010).

[30] M. G. Kanatzidis, Chem. Mater. 22, 648 (2010).

[31] G. Zhang, B. Kirk, L. A. Jauregui, H. Yang, X. Xu, Y. P. Chen and Y. Wu, Nano Lett. 12, 56 (2012).

[32] R. Fei, A. Faghaninia, R. Soklaski, J. A. Yan, C. Lo and L. Yang, Nano Lett. 14, 6393 (2014).

[33] K. Yang, S. Cahangirov, A. Cantarero, A. Rubio and R. D'Agosta, Phys. Rev. B 89, 125403 (2014).

[34] S. Kumar and U. Schwingenschlögl, Chem. Mater. 27, 1278 (2015).

[35] S. Bhattacharyya,T. Pandey and A. K. Singh, Nanotechnology 25, 465701 (2014).

[36] K. X. Chen, X. M. Wang, D. C. Mo and S. S. Lyu, J. Phys. Chem. C 2015, 119, 26706 (2015). 
[37] M. Tahir and U. Schwingenschlögl, New Journal of Physics 16, 115003 (2014).

[38] A. Arab and Q. Li, Sci. Rep. 5, 13706 (2015).

[39] S. D. Guo, arXiv:1602.03632.

[40] P. Hohenberg and W. Kohn, Phys. Rev. 136, B864 (1964); W. Kohn and L. J. Sham, Phys. Rev. 140, A1133 (1965).

[41] P. Blaha, K. Schwarz, G. K. H. Madsen, D. Kvasnicka and J. Luitz, WIEN2k, an Augmented Plane Wave + Local Orbitals Program for Calculating Crystal Properties (Karlheinz Schwarz Technische Universität Wien, Austria) 2001, ISBN 3-9501031-1-2

[42] J. P. Perdew, K. Burke and M. Ernzerhof, Phys. Rev. Lett. 77, 3865 (1996).

[43] A. H. MacDonald, W. E. Pickett and D. D. Koelling, J. Phys. C 13, 2675 (1980).

[44] D. J. Singh and L. Nordstrom, Plane Waves, Pseudopotentials and the LAPW Method, 2nd Edition (Springer, New York, 2006).

[45] J. Kunes, P. Novak, R. Schmid, P. Blaha and K. Schwarz, Phys. Rev. B 64, 153102 (2001).

[46] D. D. Koelling, B. N. Harmon, J. Phys. C Solid State Phys. 10, 3107 (1977).

[47] G. K. H. Madsen and D. J. Singh, Comput. Phys. Commun. 175, 67 (2006).

[48] B. L. Huang and M. Kaviany, Phys. Rev. B 77, 125209 (2008).

[49] L. Q. Xu, Y. P. Zheng and J. C. Zheng, Phys. Rev. B 82,
$195102(2010)$.

[50] J. J. Pulikkotil, D. J. Singh, S. Auluck, M. Saravanan, D. K. Misra, A. Dhar and R. C. Budhani, Phys. Rev. B 86, 155204 (2012).

[51] H. L. Zhuang and R. G. Hennig, J. Phys. Chem. C 117, 20440 (2013).

[52] G. B. Liu, W. Y. Shan, Y. Yao, W. Yao and D. Xiao, Phys. Rev. B 88, 085433 (2013).

[53] G. B. Liu, D. Xiao, Y. G. Yao, X. D. Xu and W. Yao, Chem. Soc. Rev. 44, 2643 (2015).

[54] T. J. Scheidemantel, C. Ambrosch-Draxl, T. Thonhauser, J. V. Badding, J. O. Sofo, Phys. Rev. B 68, 125210 (2003).

[55] G. K. H. Madsen, J. Am. Chem. Soc. 128, 12140 (2006).

[56] X. Gao, K. Uehara, D. Klug, S. Patchkovskii, J. Tse, T. Tritt, Phys. Rev. B 72, 125202 (2005).

[57] D. Parker, X. Chen and D. J. Singh, Phys. Rev. Lett. 110, 146601 (2013).

[58] L. H. Wu, J. Yang, S. Y. Wang, P. Wei, J. H. Yang, W. Q. Zhang and L. D. Chen, Phys. Rev. B 90, 195210 (2014).

[59] W. Liu, X. J. Tan, K. Yin, H. J. Liu, X. F. Tang, J. Shi, Q. J. Zhang and C. Uher Phys. Rev. Lett. 108, 166601 (2012).

[60] W. Yao, E. Y. Wang et al., arXiv:1603.02140.

[61] Kevin F. Garrity, arXiv:1601.01622.

[62] Y. L. Wang et al., Nano Lett. 15, 4013 (2015). 